\title{
Impresión 3D y sus beneficios en el campo de la educación médica, entrenamiento y asesoría del paciente.
}

\section{D printing and its benefits in the field of medical education, training and patient counseling.}

\author{
Alexander Heinze ${ }^{1,2,3}$, Mario Basulto-Martinez ${ }^{4,5}$, Rodrigo Suárez-Ibarrola ${ }^{6}$ \\ 1 American British Cowdray Medical Center, Ciudad de México; https://orcid.org/0000-0002- \\ 2164-656X; alexander@heinzemg.com; \\ 2 Facultad de Medicina, Universidad Nacional Autónoma de México, Ciudad de México \\ 3 Departamento de Urología, Marienkrankenhaus, Hamburg, Alemania \\ 4 Centro Europea de Entrenamiento en Endourología, Departamento de Urología, Ospedale \\ San Raffaele - Turro, Milán, Italia. https://orcid.org/0000-0001-6239-9078 \\ 5 Departamento de Urología, Hospital Regional de Alta Especialidad de la Península de \\ Yucatán, Mérida, México. \\ 6 Departamento de Urología, Hospital Universitario de Friburgo, Alemania; \\ https://orcid.org/0000-0001-5725-8780 \\ * Correspondencia: alexander@heinzemg.com
}

Recibido: 1 de abril de 2020; Aceptado: 4 de abril de 2020; Publicado: 4 de abril de 2020

Resumen: La impresión digital en 3D ha adquirido una gran importancia en muchas áreas, incluida la medicina y, en particular, la cirugía. Es una técnica que permite la producción de modelos a partir de imágenes bidimensionales con la ayuda de un software especializado y una impresora 3-D. Las aplicaciones pueden ser innumerables pero su uso se ha centrado en la educación médica, la planificación de la cirugía, la asesoría de los pacientes y la creación de prótesis o injertos de tejidos. El objetivo de este texto es proporcionar al lector una visión general de las aplicaciones disponibles actualmente, así como de las líneas de investigación más prometedoras para el futuro cercano.

Palabras clave: Impresión tridimensional; Impresión 3d; Educación Médica; Tecnología Médica; Asesoría de pacientes

\begin{abstract}
Digital 3D printing has become very important in many areas, including medicine and, in particular, surgery. It is a technique that allows the production of models from two-dimensional images with the help of specialized software and a 3-D printer. The applications can be innumerable, but their use has focused on medical education, surgery planning, patient counseling, and the creation of prostheses or tissue grafts. The objective of this text is to provide the reader with an overview of the applications currently available, as well as the most promising lines of research for the near future.
\end{abstract}

Keywords: 3-Dimensional Printing; 3-D Printing; Medical Education; Medical technology; Patient Counseling

\section{Introducción}

La impresión en 3D ha despertado un gran interés $\mathrm{y}$, aunque no se trata de un descubrimiento nuevo, su aplicación ha crecido exponencialmente en las últimas décadas. Sus orígenes se inician a finales de los años ochenta y reconocen a Charles 
Hull como el inventor y fundador de la impresión en tercera dimensión, pero fue hasta la década de los noventa cuando cobró importancia en el campo de la medicina $(1,2)$.

La técnica parte del principio de lo que se conoce como impresión aditiva; en la que la que el objeto deseado se construye depositando múltiples capas de material una encima de la otra. Se trata de un proceso altamente especializado capaz de convertir imágenes digitales en modelos físicos tridimensionales que pueden tener diversos usos. Entre ellos la creación de modelos didácticos para mejorar la comprensión de la anatomía en el caso de estudiantes o residentes, para planificar procedimientos quirúrgicos en el caso de cirujanos, como útiles herramientas durante el asesoramiento los pacientes previo a una cirugía y la elaboración de injertos o prótesis.

Las impresoras digitales utilizan comúnmente distintos polímeros o plásticos para elaborar los modelos, pero cabe mencionar que existen impresoras capaces de trabajar con otros materiales incluyendo metales, nylon e incluso geles biológicos.

Las primeras aplicaciones de esta tecnología fueron en campos como la ortopedia, la cirugía plástica y la odontología, pero ahora la técnica de impresión 3D ha aumentado considerablemente sus aplicaciones. Ha demostrado su en utilidad muchas otras especialidades, e incluso siendo atractivo en aspectos económicos de la salud para la reducción de tiempos quirúrgicos y el número de días de estancia intrahospitalaria $(3,4)$.

En un principio los modelos solían estar hechos de materiales rígidos y carecían de realismo y detalles anatómicos, pero la introducción de nuevas materias primas ha permitido crear modelos de materiales más elásticos similares al tejido humano que, con la adición de colores y otros detalles, han dado lugar a modelos con un gran realismo.

\section{Escaneo y procesamiento de modelos}

Los modelos anatómicos 3D suelen crearse a partir de imágenes de archivos médicos, más comúnmente DICOM (Digital Imaging and Communications in Medicine), que son obtenidas ya sea de tomografías computarizadas (CT), resonancias magnéticas (MRI), angiografías $\mathrm{y}$, en el menor de los casos, ultrasonidos (USG). También es posible la creación de modelos utilizando programas de diseño como CAD (computer aided design).

El procesamiento de imágenes requiere el uso de programas informáticos especializados; existen muchas alternativas, algunas de ellas gratuitas y otras que requieren la compra de una licencia; entre las más populares podemos mencionar Mimics (Materialise, Bélgica), Compact View III (Optimum Solutions, Corea) y Blender (Blender foundation, Holanda). Durante el llamado "procesamiento" de las imágenes se busca mantener sólo las estructuras de interés y se eliminará el resto. Se trata de un proceso que puede consumir mucho tiempo y que requiere personal capacitado, por lo que es altamente recomendable que este paso sea supervisado por radiólogos experimentados (2). Aunque ya existen programas que realizan el procesamiento de imágenes de forma automática, los autores de éste texto recomendamos, hasta ahora, el procedimiento manual por expertos para obtener la mayor precisión posible (figura $1)$. 


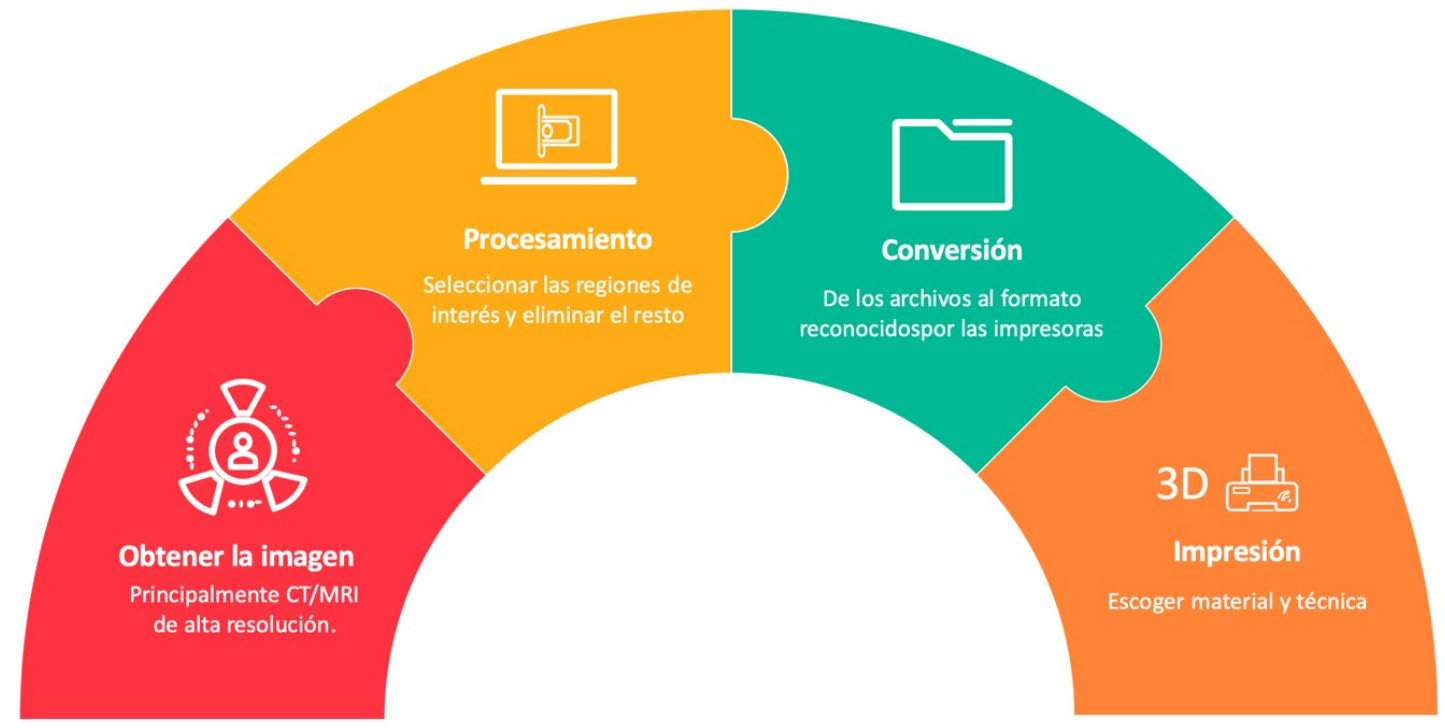

Figura 1. Fases del proceso de impresión 3D

Una vez procesadas las estructuras de interés, los archivos deben ser convertidos a un formato de impresión. Comúnmente se utiliza el lenguaje "STL" (Standard Tessellation Language) que es capaz de ser reconocido por las impresoras 3D. Este formato tiene la característica de trabajar utilizando figuras geométricas, principalmente triángulos, para cubrir completamente la superficie de los modelos (1).

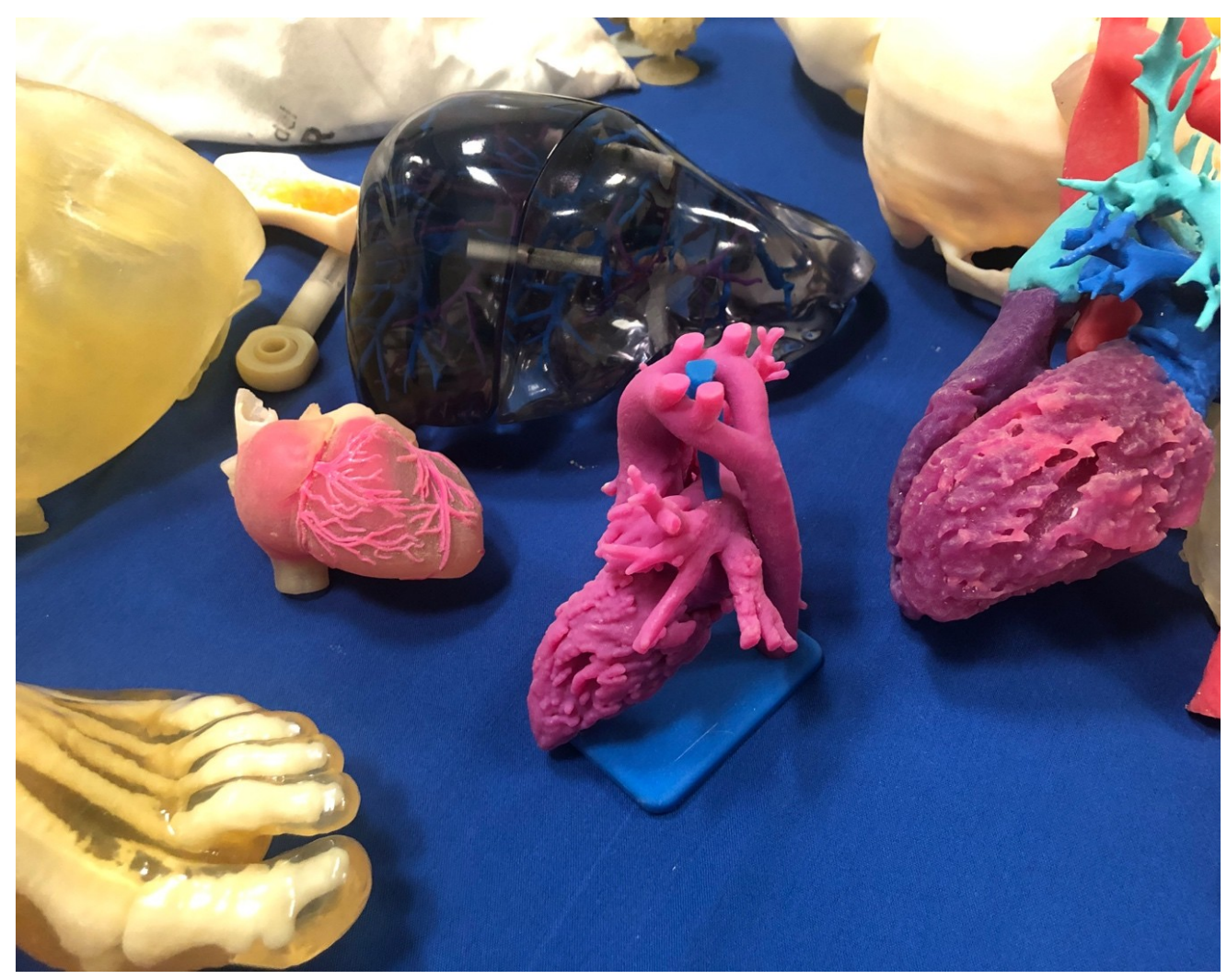

Figura 2. Modelos obtenidos por impresión 3D

\section{Impresión}

Finalmente, se imprimirá el modelo (figura 2). En cuanto a las tecnologías de impresión en 3D disponibles, la más común es la llamada FDM (Fused Deposition 
Modelling), aunque hay otras como SLS (selective laser sintering) o SLA (stereolithography) $(5,6)$.

\section{Aplicaciones en la educación y entrenamiento médico}

En las últimas décadas ha aumentado el interés por el entrenamiento quirúrgico, especialmente el que se centra en la repetición de tareas, con objetivos previamente definidos y realizado dentro de escenarios controlados. Es aquí donde los modelos 3D podrían ser particularmente útiles. Ya sea dentro de hospitales de enseñanza, o bien en centros de entrenamiento donde se preparan a los residentes y futuros cirujanos, los modelos 3D podrían ser una solución para dotar a los aprendices de una extensa variedad de modelos diseñados para el desarrollo, mejora y/o mantenimiento de habilidades quirúrgicas.

Pueden se útiles desde las escuelas de medicina hasta el posgrado. Hakmin Lee creó modelos 3D de tumores renales que fueron entregados a un grupo de estudiantes de medicina para evaluar su utilidad como herramienta para reconocer el sitio en donde se encontraban la lesión. Sorprendentemente, los estudiantes de medicina fueron capaces de determinar la ubicación exacta de los tumores con la ayuda de los modelos 3D en un 70\%, por el contrario, cuando sólo tenían las imágenes computarizadas lo hicieron en un 47,3\%. Este autor también demostró que los modelos fueron de gran utilidad para médicos especialistas quienes respondieron un cuestionario (7) reconociendo ventajas para una mejor comprensión de la anatomía $(8.9 / 10)$, la planificación quirúrgica $(8.2 / 10)$ y la localización del tumor $(8.4 / 10)$ especialmente en aquellos tumores de tipo endofíticos (9.5/10).

\section{Planificación quirúrgica}

La nefrectomía parcial ha adquirido un gran auge en las últimas décadas, ya que su objetivo es obtener una mayor preservación del parénquima y, por lo tanto, de la función renal. En general, la decisión de realizar una nefrectomía radical o una nefrectomía parcial se basa en la complejidad del tumor, medida de manera objetiva con ayuda de las escalas existentes, por ejemplo, R.E.N.A.L. Padua y una menos popular C-Index (8-10). Estos instrumentos han permitido mejorar la clasificación de los tumores, pero a veces son insuficientes para conocer en detalle la anatomía y la relación del tumor con las estructuras adyacentes, incluidas las venas, las arterias y otros órganos.

La impresión digital ha permitido crear modelos que pueden ser analizados y evaluados por todos en el equipo para una correcta planificación quirúrgica. Estos modelos podrían ser especialmente útiles en aquellos pacientes con anatomía compleja, donde hay múltiples vasos, en pacientes con enfermedad renal crónica (ERC) previa y/o en casos con tumores renales muy grandes (11).

Por lo general, se obtienen inicialmente imágenes digitales (DICOM) que luego son segmentadas y procesadas por expertos que seleccionan las regiones de interés (ROI) y finalmente cada componente se imprime por separado. Los modelos impresos parecen ofrecer una mayor comprensión de la anatomía, como lo demostró Wake (12), quien presentó una serie de casos oncológicos a distintos cirujanos. El autor en la primera reunión presentó los casos con ayuda de imágenes digitales (PACS). Posteriormente, en una reunión distinta, se incluyó un modelo 3D como complemento a las imágenes digitales. Finalmente, los cirujanos respondieron a un cuestionario preoperatorio que incluía decisiones quirúrgicas.

Fue posible registrar además los datos de las notas operatorias de los pacientes presentados. Las respuestas obtenidas en la sesión con los modelos tridimensionales 
permitieron predecir el 100\% de los casos que tipo de cirugía se realizó (nefrectomía parcial o una nefrectomía radical), en comparación con el 92,6\% cuando únicamente se tenían imágenes digitales. Lo mismo ocurrió con el abordaje abierto vs. laparoscópico, transperitoneal vs. retroperitoneal y la ligadura de vasos, donde el uso del modelo 3D predijo lo que ocurrió en la cirugía en un 81,5\%, 55,6\% y 85,3\% en comparación con la imagen convencional en un 77,8\%, 59,3\% y 81,5\% respectivamente. En general, todos los cirujanos consideraron útil utilizar modelos 3D para comprender mejor la anatomía y planificar correctamente el procedimiento. Se informó que los costos de cada modelo eran de alrededor de 1000 dólares (usd) y el tiempo de impresión era de 10 horas con 7 horas de procesamiento de imágenes (13).

Otro aspecto que hay que tener en cuenta es que el uso de modelos tridimensionales en la planificación quirúrgica disminuye los tiempos de isquemia y el número de complicaciones. Gang Fan (14) trabajó con 127 pacientes a los que se les realizó una nefrectomía parcial laparoscópica que dividió en dos grupos, en 69 de ellos realizó un modelo 3D antes de la cirugía para favorecer la navegación y la planificación preoperatoria, en el otro grupo se sometió a una LPN convencional sin modelo. Demostraron que los casos en los que se fabricó un modelo 3D preoperatorio tuvieron en general menos tiempo de isquemia caliente (WIT) así como una menor pérdida de sangre intraoperatoria estimada (EIBL) en aquellos pacientes con tumores complejos definidos con R.E.N.A.L. score $\geq 8$ Los autores informaron de un coste por modelo de aproximadamente 500 usd.

\section{Biopsias de próstata}

Wang desarrolló un modelo tridimensional como asistente cognitivo en la biopsia de próstata. Los pacientes se sometieron a una resonancia magnética antes de la biopsia donde las lesiones fueron localizadas por dos radiólogos diferentes. Usando imágenes DICOM, estas fueron convertidas a un formato STL para su impresión. El modelo fue otorgado al cirujano previo a la biopsia de próstata. Se realizó una biopsia de próstata sistemática estándar de 12 cilíndros, adicionalmente se obtuvieron 2-3 cilindros de áreas sospechosas en la resonancia magnética. Se incluyeron un total de 16 pacientes, 10 de los cuales fueron diagnosticados con cáncer de próstata. Los resultados fueron muy interesantes debido a que: de los 10 pacientes con cáncer de próstata, 7 pacientes tuvieron tanto biopsias sistemáticas como biopsias dirigidas positivas, 1 fue diagnosticado por biopsia sistemática solamente, y 2 tuvieron biopsia dirigida positiva solamente. Esto quiere decir que la probabilidad de obtener una biopsia positiva fue del $46,2 \%$ en la biopsia dirigida, en comparación con el $22,4 \%$ de la biopsia sistemática convencional. En este estudio, el uso de modelos 3D como asistente cognitivo podría traducirse en un método más efectivo capaz de mejorar el diagnóstico del cáncer de próstata (15).

\section{Ensayo de la cirugía}

Von- Rundstedt y otros describieron la creación de un modelo de silicona a partir de imágenes obtenidas por TAC o resonancia magnética y utilizando un software llamado 3D Slicer (https://www.slicer.org/). El procedimiento permitió al cirujano un entrenamiento previo con un modelo idéntico al de la cirugía real.

El procedimiento simulado fue video-grabado y se determinó que los márgenes eran negativos cuando no había restos de color del tumor en el lecho de resección. Estos resultados se registraron para su posterior análisis. Durante la cirugía real el tumor fue medido volumétricamente para conocer la cantidad de tejido resecado.

Se compararon tanto el volumen de tumor resecado, con el volumen de material resecado durante el ensayo con el modelo de silicona y el volumen calculado de las 
imágenes digitales. Se concluyó que no había una diferencia significativa al comparar los tres volúmenes de los 10 pacientes. Además, los autores recomendaron registrar el tiempo de la escisión del tumor en el modelo de silicona ya que guardaba una relación con el tiempo total de la isquemia en el paciente real (16).

\section{Impresión digital de los injertos}

La impresión digital ha tenido otras aplicaciones en el campo clínico urológico, muchas de ellas con un futuro prometedor como la impresión de implantes biológicos.

La estenosis uretral es una patología con una alta incidencia, se estima que en los EE.UU. hay entre 1,5 millones de consultas por año y afecta hasta el $0,6 \%$ de las personas en riesgo. Es una patología que afecta gravemente a la calidad de vida del paciente (17). Hasta ahora se habían utilizado injertos autólogos, principalmente de la mucosa oral, para el tratamiento de las estenosis largas, pero con la llegada de la impresión en 3D esto podría cambiar. Zhang y sus colaboradores han estado trabajando en modelos de bioimpresión con los que han podido recrear una estructura similar a la de la uretra con células uroteliales y de músculo liso. Los autores crearon una "tinta biológica" que contiene fibrina, gelatina y ácido hialurónico. La combinación de elementos biológicos y sintéticos garantizaba la estabilidad y la resistencia mecánica por un lado y además fue posible demostrar la proliferación del tejidos en un modelo animal mediante (18).

\section{Asesoramiento al paciente}

Como se mencionó en una sección anterior, la creación de modelos personalizados puede ser útil para explicar a los pacientes el procedimiento al que se someterán.

Como prueba de ello se realizaron entrevistas con aplicación de un cuestionario basal a pacientes en la que se le dio información general sobre el procedimiento y se le mostraron imágenes digitales convencionales. Posteriormente se les entregó un modelo personalizado y se le aplicó nuevamente el cuestionario. Cuando se compararon los resultados de ambas pruebas, los pacientes dijeron que tenían una mejor comprensión de la anatomía, el procedimiento planificado y una mejor comprensión de las posibles complicaciones después de mirar el modelo.

Los cuestionarios finales (después del uso del modelo 3D) mostraron una mejora en las secciones relacionado con la anatomía $60 \%(\mathrm{p}=0,017)$, el procedimiento quirúrgico planificado en un $60 \%(\mathrm{p}=0,017)$, y la comprensión de las complicaciones relacionadas con la cirugía en un $64 \%(p=0,015)$. En este estudio, cada modelo costó aproximadamente 100 dólares por modelo y se tardó 2 días en fabricarlo (19).

\section{Dispositivos médicos}

Del Junco y otros han descrito recientemente la impresión de catéteres, los autores también informan del éxito de la impresión de trocares utilizados en modelos de cerdos de manera satisfactoria y abren un debate sobre las posibilidades de futuro (20). Se espera que haya un aumento considerable en el uso de este tipo de instrumentos, así como en el número de aplicaciones que puedan surgir.

\section{Costes y materiales}

Aunque una de las limitaciones para adoptar esta nueva tecnología es el coste, los autores están seguros de que disminuirá con la llegada de nuevas impresoras y materiales más accesibles. Los polímeros representan los materiales más accesibles en comparación con los metales o los bioelementos como la matriz extracelular que 
son por el momento mucho más caros. Sin embargo, también se abrió la necesidad de hacer compatibles otros procesos hospitalarios, como la esterilización, para poder adoptar masivamente modelos hechos de polímero en la práctica clínica (5).

Un área de oportunidad podría ser la impresión de los dispositivos médicos dentro de los hospitales. Esta posibilidad puede reducir el costo de transporte y almacenamiento de los dispositivos médicos al disponer de impresoras 3D capaces de producir instrumentos de uso instantáneo. Además, existe también la posibilidad de actualizar los instrumentos con sólo la adquisición de actualizaciones de los archivos existentes (21).

\section{Conclusiones}

- Hasta ahora el campo quirúrgico se había apoyado en imágenes en 2 dimensiones y a menudo en reconstrucciones digitales en 3D, con la llegada de la impresión digital en 3D se abren nuevos horizontes que podrían hacer más sencillos y seguros los procedimientos.

- Se trata de una tecnología que cambiará las prácticas actuales de enseñanza para estudiantes y residentes, asesoramiento de pacientes, técnica quirúrgica y producción de dispositivos médicos que conocemos.

- A medida que se convierte en una tecnología cada vez más disponible, se espera que los beneficios pronto superen a los costes de fabricación. El futuro ya está aquí.

Material suplementario: no hay.

Financiación: No ha habido financiación.

Agradecimientos: Agradecemos a la Lic M.I. Calzada-Zubiría por el apoyo de las ilustraciones en la realización de este documento.

Declaración de conflicto de interés: Los autores declaran no tener ningún conflicto de interés.

\section{Referencias}

1. Parikh N, Sharma P. Three-Dimensional Printing in Urology: History, Current Applications, and Future Directions. Urology. 2018;121:3-10. doi:10.1016/j.urology.2018.08.004

2. Smith, B., Dasgupta, P. 3D printing technology and its role in urological training. World J Urol (2019). doi: 10.1007/s00345-019-02995-1

3. Pornrattanamaneewong C, Chareancholvanich K, Narkbunnam R. A prospective randomised controlled study of patient-specific cutting guides compared with conventional instrumentation in total knee replacement. Bone Joint J. 2013;95-B(3):354-359. doi:10.1302/0301-620X.95B3.29903

4. Özgür BC, Ayylldız A. 3D Printing in urology: Is it really promising? Turk J Urol. 2018;44(1):6-9. doi:10.5152/tud.2018.20856

5. Manning TG, O'Brien JS, Christidis D, et al. Three dimensional models in uro-oncology: a future built with additive fabrication. World J Urol. 2018;36:557-563. doi:10.1007/s00345018-2201-2

6. Özgür BC, Ayyıldız A. 3D printing in urology: is it really promising? Turkish J Urol. 2018;44(1):6-9. doi:10.5152/tud.2018.20856

7. Lee H, Nguyen NH, Hwang S Il, Lee HJ, Hong SK, Byun S-S. Personalized 3D kidney model produced by rapid prototyping method and its usefulness in clinical applications. Int Braz J Urol. 2018;44(5):952-957. doi:10.1590/S1677-5538.IBJU.2018.0162

8. Kutikov A, Uzzo RG. The R.E.N.A.L. Nephrometry Score: A Comprehensive Standardized System for Quantitating Renal Tumor Size, Location and Depth. J Urol. 2009;182(3):844853. doi:10.1016/j.juro.2009.05.035 
9. Ficarra V, Novara G, Secco S, et al. Preoperative Aspects and Dimensions Used for an Anatomical (PADUA) Classification of Renal Tumours in Patients who are Candidates for Nephron-Sparing Surgery. Eur Urol. 2009;56(5):786-793. doi:10.1016/j.eururo.2009.07.040

10. Simmons MN, Ching CB, Samplaski MK, Park CH, Gill IS. Kidney Tumor Location Measurement Using the C Index Method. J Urol. 2010;183(5):1708-1713. doi:10.1016/j.juro.2010.01.005

11. Westerman ME, Matsumoto JM, Morris JM, Leibovich BC. Three-dimensional Printing for Renal Cancer and Surgical Planning. Eur Urol Focus. 2016;2(6):574-576. doi:10.1016/j.euf.2016.12.009

12. Wake N, Rude T, Kang SK, et al. 3D printed renal cancer models derived from MRI data: application in pre-surgical planning. Abdom Radiol. 2017;42(5):1501-1509. doi:10.1007/s00261-016-1022-2

13. Westerman ME, Matsumoto JM, Morris JM, Leibovich BC. Clinical Consultation Guide Three-dimensional Printing for Renal Cancer and Surgical Planning. Eur Urol Focus. 2016;2(6):574-576. doi:10.1016/j.euf.2016.12.009

14. Fan G, Meng Y, Zhu S, et al. Three-dimensional printing for laparoscopic partial nephrectomy in patients with renal tumors. J Int Med Res. 2019;47(9):4324-4332. doi:10.1177/0300060519862058

15. Wang $Y$, Gao X, Yang Q, et al. Three-dimensional printing technique assisted cognitive fusion in targeted prostate biopsy. Asian J Urol. 2015;2:214-219. doi:10.1016/j.ajur.2015.09.002

16. Von Rundstedt F-C, Scovell JM, Agrawal S, Zaneveld J, Link RE. Utility of patient-specific silicone renal models for planning and rehearsal of complex tumour resections prior to robot-assisted laparoscopic partial nephrectomy HHS Public Access. BJU Int. 2017;119(4):598-604. doi:10.1111/bju.13712

17. Latini JM, Mcaninch JW, Brandes SB, Chung JY, Rosenstein D. SIU/ICUD consultation on Urethral Structures: Epidemiology, Etiology, Anatomy, and Nomenclature of Urethral Stenoses, Structures, and Pelvic Fracture Urethral Disruption Injuries.Urology. 2014 Mar;83(3 Suppl):S1-7. doi:10.1016/j.urology.2013.09.009

18. Zhang $\mathrm{K}, \mathrm{Fu} \mathrm{Q}$, Yoo J, et al. 3D bioprinting of urethra with PCL/PLCL blend and dual autologous cells in fibrin hydrogel: An in vitro evaluation of biomimetic mechanical property and cell growth environment. Acta Biomater. 2017;50:154-164. doi:10.1016/j.actbio.2016.12.008

19. Atalay HA, Canat HL, Ülker V, Alkan İ, Özkuvanci Ü, Altunrende F. Impact of personalized three-dimensional (3D) printed pelvicalyceal system models on patient information in percutaneous nephrolithotripsy surgery: a pilot study. Int Braz J urol. 2017;43:470-475. doi:10.1590/S1677-5538.IBJU.2016.0441

20. Del Junco M, Okhunov Z, Yoon R, et al. Development and initial porcine and cadaver experience with three-dimensional printing of endoscopic and laparoscopic equipment. J Endourol. 2015;29(1):58-62. doi:10.1089/end.2014.0280

21. Youssef RF, Spradling $\mathrm{K}$, Yoon $\mathrm{R}$, et al. Applications of three-dimensional printing technology in urological practice. BJU Int. 2015;116(5):697-702. doi:10.1111/bju.13183

(C) 2020 por los autores. Enviado para su posible publicación en acceso abierto bajo los términos y condiciones de la licencia Creative Commons Attribution (CC BY) (http://creativecommons.org/licenses/by/4.0/). 